Purdue University

Purdue e-Pubs

Purdue CIBER Working Papers

Krannert Graduate School of Management

$1-1-2000$

\title{
Global Production Planning under Exchange-Rate Uncertainty
}

Maqbool Dada

Purdue University

Herbert Moskowitz

Purdue University

Burak Kazaz

University of Miami

Follow this and additional works at: http:// docs.lib.purdue.edu/ciberwp

Dada, Maqbool; Moskowitz, Herbert; and Kazaz, Burak, "Global Production Planning under Exchange-Rate Uncertainty" (2000). Purdue CIBER Working Papers. Paper 9.

http://docs.lib.purdue.edu/ciberwp/9

This document has been made available through Purdue e-Pubs, a service of the Purdue University Libraries. Please contact epubs@purdue.edu for additional information. 


\title{
Global Production Planning under Exchange-Rate Uncertainty
}

\author{
Maqbool Dada
}

Purdue University

\section{Herbert Moskowitz}

Purdue University

\author{
Burak Kazaz
}

University of Miami

2000-009

Krannert Graduate School of Management

Purdue University

1310 Krannert Building

West Lafayette, IN $47907-1310$

(765) 494-4463

Fax (765) 494-9658 


\title{
Global Production Planning under Exchange-Rate Uncertainty
}

\author{
Burak Kazaz ${ }^{1 \& 2}$ Maqbool Dada ${ }^{3}$ Herbert Moskowitz ${ }^{3}$ \\ ${ }^{1}$ School of Business, University of Miami, 414 Jenkins Building, Coral Gables, FL 33124 USA \\ 2 IBM T. J. Watson Research Center, Yorktown Heights, NY 10538, USA \\ ${ }^{3}$ Krannert Graduate School of Management, Purdue University, W. Lafayette, IN 47907, USA
}

Draft: October 11, 2001

\begin{abstract}
Motivated by a production planning problem in an actual global manufacturing network, we examine the impact of exchange-rate uncertainty ... namely the volatility in and correlations among exchange rates ... on the choice of various optimal production policies and the conditions which lead to them. A two-stage stochastic program with recourse is developed that provides opportunities to hedge financial risk by allowing for some production planning decisions to be deferred until exchange rates are realized. The proposed model and analysis yield the following results: (1) Under significant exchange-rate uncertainty it may be optimal to hedge production by producing less than total demand, (2) production hedging is more likely an optimal policy when either the profit margin is small and/or the exchange rates are highly volatile, (3) the probability of following a production hedging policy decreases as the correlations between exchange rates monotonically increase in a positive direction, (4) after exchange rates are realized it may be optimal to hedge allocation by not allocating all production to some of the markets, and (5) given production and/or allocation hedging, our model identifies the value of financial hedging for each firm rather than a single option price for all firms. This guarantees that all markets are served and that each firm gets an option price that is commensurate with its exchange-rate risk. These findings and implications are illustrated with examples adapted from data provided by IBM.
\end{abstract}




\section{Introduction}

This study is motivated by a production-planning problem regularly faced by a global electronics manufacturer, and common to many global companies. The component manufacturing division of the company periodically receives projected demand from the company's personal computer (PC) division as well as other original equipment manufacturers (OEM). These projections are crucial to determining the quarterly aggregate plan that allocates how much to manufacture of each product in each of its plants in order to maximize expected profit. Along with setting the production plan, the company allocates budgets and sets transfer prices for sales to all countries. Consequently, the revenue of the manufacturing division is subject to financial risk from currency conversion since exchange rates fluctuate over time.

The standard approach as detailed in Flaherty's [1996] case, Applichem (A), is to replace those parameters that are influenced by fluctuations in exchange rates by their expected values. She then proceeds to solve an aggregate production-planning problem in much the same way as when the problem setting is domestic. Since all production and allocation decisions are made before the realization of exchange rates, this approach has the limitation that it does not take into account that in practice some decisions, for example the allocation decision, may be deferred until exchange rates are realized. Consequently, using this approach foregoes significant opportunities to hedge financial risk from exchange-rate fluctuations by allowing additional information prior to determining an allocation policy.

This motivates us to focus on developing a modeling approach that provides opportunities to mitigate financial risk by explicitly allowing for some decisions to be deferred until the exchange rates are realized. It leads naturally to the formulation of a stochastic program with recourse whose objective is to maximize expected profit. In the first stage of the problem, given projected demands in each country and costs for an aggregate product, the production plan is determined. A hedge is introduced by allowing planned production to be less than the total demand. After the first stage decisions are made, the realized exchange rates are observed. Given the realized exchange rates, in the second or recourse stage of the problem, production is allocated to meet demand. A second hedge is introduced here by providing the opportunity to discard production if some exchange-rate realizations are deemed to be economically unfavorable.

Our model differs considerably from related models in the literature because it advocates two forms of operational hedging to mitigate exchange-rate risk. In one form, it can be optimal to produce less than the total demand (stage 1). We coin this as production hedging. The optimality of production hedging holds true notwithstanding the assumption that all foreign markets are ex-ante profitable (i.e. expected revenue is greater than the total of manufacturing and transportation costs), making it a powerful result. In the other form, it can be optimal to discard some production if the realized exchange rates are so unfavorable that revenues do not justify transportation expenses. We call this allocation hedging (stage 2 or recourse stage). One might 
intuit that allocation hedging leads to production hedging. Although allocation hedging helps to contribute to this strategy, production hedging can be optimal without the presence of allocation hedging. This observation further enhances the value of production hedging. Therefore, an optimal solution can have none, one, or both of these two hedging features. We also quantify the magnitude of the financial risk faced by a manufacturer who is compelled to meet all of the demand in its aggregate plan. This is determined by appropriately comparing the optimal solution of our program with that of suitably related model variants. This analysis allows us to compute reservation prices for any financial instrument (i.e. the maximum amount of money that one should be willing to pay for such instruments) that may be purchased to hedge risk. Finally, our analysis also shows how the financial risk can be appropriately shared among different functional entities within the company.

Section 2 provides a review of the operational hedging literature. We begin our analysis in Section 3 with the most general form of our model. Then, considering several simpler variants enables us to present virtually all the structural results pertinent to this model, characterize the optimal policy structure, and develop managerial insights. Exchange-rate uncertainty arises in our model from the following two sources: (a) the natural volatility in each real (not nominal) exchange rate relative to the numeraire currency; and (b) the correlation among the exchange rates when revenues arise from two or more foreign markets where the revenue from each is denominated in its own local currency. In section 4, we apply our model to the global manufacturing network of IBM, described in our motivating example. Concluding remarks are discussed in Section 5. Finally, proofs are presented in an addendum available from the authors.

\section{Literature Review}

To put our two uses of operational hedges in perspective, consider the natural sequence of decisions in managing a manufacturing network: 1) invest in capacity, 2) manufacture, and 3) after manufacturing occurs, allocate production to meet demand. Exchange rates are known either after steps 1,2 or 3 . This leads naturally to thinking of the problem as a stochastic program with recourse. Figure 1 shows a framework to classify modeling approaches for these conditions. For the global electronics manufacturer who motivated this study, the exchange rates are realized after step 2 so that only the allocation decisions are made in the recourse stage of the problem. We will refer to this problem as the recourse-after-production problem. Obviously, if exchange rates are known prior to all three manufacturing steps, then we can obtain the value of perfect information as a benchmark case. Finally, in the traditional domestic analogue of our problem, exchange rates are realized after step 3 . Thus, there is no opportunity for recourse, and random exchange rates can be replaced by their expected values. As a general rule, the more decisions that are deferred till exchange rates are realized, the higher the resulting expected contribution. While the modeling approach developed in this paper is sufficiently general to apply to all four situations outlined above, we focus on the case when exchange rates are realized between steps 2 and 3 
in order to highlight the motivating application.

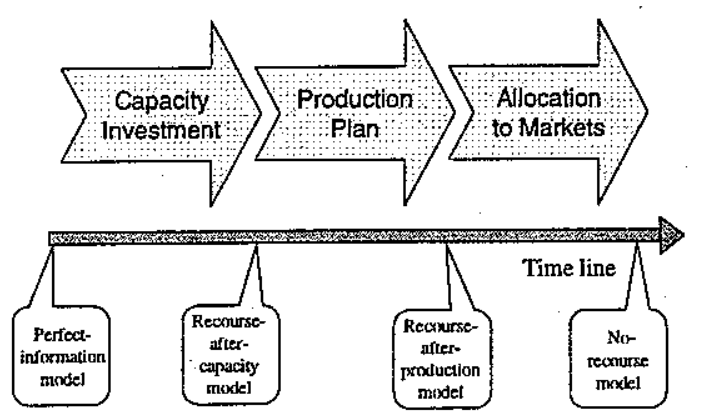

Figure 1: Natural sequence of operational decisions and models that have different timing of exchange-rate realizations

Earlier models of operational hedging have focused on the long-term, more strategic issues of capacity investment and production switching. Huchzermeier and Cohen [1996] viewed an operational hedge as flexibility to utilize a number of supply contract and production location options in a global supply chain. They employed a recourse-after-capacity model (viz, exchange rates are observed after capacity decisions are made) in selecting the design of the supply chain. Although their study does not provide structural results on the nature of the optimal policy, it presents numerical examples that show that operational hedging can reduce the downside risk of exchange rates in the longer term. The authors suggest that financial hedging be used to reduce the variability of the firm's cash flows in the short term. Using the same modeling approach, Huchzermeier [1998] evaluated the option value of operational flexibility in Applichem's network. Aspects of Huchzermeier and Cohen's [1996] research may be seen as extending Kogut and Kulatilaka's [1994] uncapacitated model of costly switching of production from one supplier to another in response to changing macroeconomic data. This switching strategy can also be interpreted as an operational hedge. Using a similar approach, Dasu and Li [1997] determined production switchover among plants when costs were influenced by exchange rates. In a more recent paper, Kouvelis et al. [2001] prescribed the choice of ownership structure (e.g. exporting, joint venture, and whollyowned subsidiary) for a set of production facilities under the presence of switchover costs. Rosenfield [1996] provided a complementary approach to that proposed by Huchzermeier and Cohen [1996]. His production and capacity decisions were based on minimizing costs influenced by exchange-rate fluctuations. In another study, Kouvelis and Gutierrez [1997] determined optimal production quantities and transfer prices within two markets where demand of each occurred in non-overlapping time periods. Exchange-rate uncertainty was also investigated in the context of supplier relationships where order quantities and contract terms were determined based on such fluctuations (e.g. Kouvelis [1999], Scheller-Wolf and Tayur [1999]).

The above models complement earlier work on incorporating exchange-rate uncertainty into the strategic 
decision of capacity investment. Jucker and Carlson [1976] were the first to incorporate exchange-rate uncertainty within the context of an uncapacitated plant location problem. They considered a model in which there is demand and price uncertainty. Uncertainty in exchange rates was embedded in the uncertainty in prices since exchange rates were assumed to influence prices in local markets. In a series of papers using a notion of an exchange-rate mean-variance trade-off, this model was extended by Hodder and Jucker [1985a, 1985b]. The extension of this approach to include limited capacity was considered by Hodder and Dincer [1986]. Hodder [1984] simplified the solution technique with a model that introduced a capital market (CAPM) approach with an exchange-rate mean-covariance objective function. These studies permitted other sources of uncertainties such as fluctuations in financial markets to reflect the definition of uncertainty. Below, as a summary, we tabulate our survey of papers under recourse-after-capacity and recourse-after-production models.

Table 1: Classification of papers with respect to modeling approaches

\begin{tabular}{|l|l|}
\hline Recourse-after-capacity models & Recourse-after-production models \\
\hline Cohen and Huchzermeier [1999] & Dasu and Li [1997] \\
Hodder [1984] & Kouvelis [1999] \\
Hodder and Jucker [1985a,b] & Kouvelis and Gutierrez [1997] \\
Hodder and Dincer [1986] & \\
Huchzermeier [1998] & \\
Huchzermeier and Cohen [1996] & \\
Kogut and Kulatilaka [1994] & \\
Rosenfield [1996] & \\
Scheller-Wolf and Tayur [1999] & \\
Kouvelis et al. [2001] & \\
\hline
\end{tabular}

\section{The Model and Structural Results}

This section presents a two-stage stochastic programming model for the aggregate production planning problem of a multinational company. We first introduce the most general form of the model, then use simpler variants of the model in order to highlight key properties affecting operating decisions. The multinational company obtains its revenues in the currency of the country of sale and converts it to the home country currency by using exchange rates. Since exchange rates fluctuate, these cash flows are subject to risk. One optimal solution suggests that under certain circumstances it is better to produce less than the total projected demand. This can be viewed as a signal to inform managers that foreign prices are not properly aligned to compensate for the exchange-rate risk, albeit, it may not be possible for the manager to increase prices for competitive reasons. 
When this is the case, we determine the value of deploying financial hedging. We also consider the impact of correlated exchange rates and the timing of exchange rate realization on aggregate production planning.

\subsection{The General Model}

The first stage can be viewed as a contract to produce a certain output. The company commits to production quantities at each plant, and headquarters transfers the associated amount of working capital to plants (countries) for manufacturing purposes. Therefore, the manufacturing costs are paid in advance and can be defined in terms of the home country currency. Based on the output in stage 1 and the realized exchange rates, in stage 2 this output is allocated to markets in order to maximize expected profit (revenue less transportation costs). The definition of stages are as follows:

Stage 1: Determine the production quantity.

Stage 2: Allocate produced units to markets after observing the exchange rates.

Using the above sequence, the resulting formulation is a stochastic program with recourse. Recourse refers to carrying the optimal second stage decisions to the first stage in order to calculate the expected profit. We use the following notation to introduce the mathematical model:

Parameters:

$i$ : index representing country of production facility, $i=1, \ldots, I$

$j:$ index representing country of sale (or markets), $j=1, \ldots, J$

$r_{j}:$ unit revenue from a sale in market $j$ (in terms of the currency of country $j$ )

$c_{i}:$ unit cost of manufacturing in country $i$ plant (in terms of domestic currency)

$c_{i j}$ : unit transportation cost between countries $i$ and $j$ (in terms of domestic currency); to simplify exposure, we assume that $c_{i i}=0$ in the analysis

$e_{j}:$ a random variable representing the exchange rate that converts foreign currency $j$ to home country currency $\left(e_{j} \geq 0\right)$

$\left(e_{1}, \ldots, e_{j}, \ldots, e_{J}\right):$ a set of random variables representing the realization of exchange rates $e_{1}$ through $e_{J}$

$d_{j}:$ the projected demand in country (market) $j$

Stage 1 Decision Variable:

$X_{i}:$ the amount of production in the plant of country $i$ 
Stage 2 Decision Variables:

$X_{i j}$ : the amount produced in country $i$ and shipped for sale in country (market) $j$

To rule out trivial cases we impose the following:

(A.1) $r_{j} \overrightarrow{e_{j}}>c_{i}+c_{i j}, \forall i=1, \ldots, I, \forall j=1, \ldots, J$ (expected unit revenue from selling in market $j$ is greater than the sum of unit manufacturing cost in country $i$ and unit transportation cost from country $i$ to country $j$ )

where $\overline{e_{j}}$ is the mean value of the exchange-rate random variable for country $j$. Assumption (A.1) makes all markets to be ex-ante profitable. This assumptions is innocuous because the firm would probably not consider doing business in these markets if it was not to hold. Using this notation, our problem is formulated as a mathematical programming model which maximizes expected profit subject to transportation and demand constraints. The first stage problem can be written as:

$$
\begin{aligned}
(\mathrm{P} 1): & \operatorname{MAX}_{\left(X_{2}, \ldots, X_{i}, \ldots, X_{I}\right)} E\left[P\left(X_{1}, \ldots, X_{i}, \ldots, X_{I}\right)\right]= \\
& -\sum_{i=1}^{I} c_{i} X_{i}+E\left[P A\left(\left(X_{1}, \ldots, X_{i}, \ldots, X_{I}\right) \mid\left(e_{1}, \ldots, e_{j}, \ldots, e_{J}\right)\right)\right] \\
& \text { s.t. }
\end{aligned}
$$

$$
X_{i} \geq 0, \forall i=1, \ldots, I, \forall j=1, \ldots, J
$$

where $E\left[P A\left(\left(X_{1}, \ldots, X_{i}, \ldots, X_{I}\right) \mid\left(e_{1}, \ldots, e_{j}, \ldots, e_{J}\right)\right)\right]$ is the expected revenue obtained from producing the quantities of $\left(X_{1}, \ldots, X_{i}, \ldots, X_{I}\right)$ in the first stage and $P A\left(\left(X_{1}, \ldots, X_{i}, \ldots, X_{I}\right) \mid\left(e_{1}, \ldots, e_{j}, \ldots, e_{J}\right)\right)$ is the second stage optimal solution value for given $\left(X_{1}, \ldots, X_{i}, \ldots, X_{I}\right)$ units of production in each country (from stage 1 ) and realized exchange rates of $\left(e_{1}, \ldots, e_{j}, \ldots, e_{J}\right) . E[\cdot]$ is the expectation operator over $f\left(e_{1}, \ldots, e_{j}, \ldots, e_{J}\right)$, the joint probability density function (pdf).

The second stage problem, allocation of production to markets, can be written as:

$$
P A\left(\left(X_{1}, \ldots, X_{i}, \ldots, X_{I}\right) \mid\left(e_{1}, \ldots, e_{j}, \ldots, e_{J}\right)\right)=\operatorname{MAX}_{\left(X_{11}, \ldots, X_{i j}, \ldots, X_{I J}\right)} \cdot \sum_{j=1}^{J} \sum_{i=1}^{I}\left(r_{j} e_{j}-c_{i j}\right) X_{i j}
$$

s.t.

$$
\begin{aligned}
\sum_{j=1}^{J} X_{i j} & \leq X_{i}, \forall i=1, \ldots, I \\
\sum_{i=1}^{I} X_{i j} & \leq d_{j}, \forall j=1, \ldots, J \\
X_{i j} & \geq 0, \forall i=1, \ldots, I, \forall j=1, \ldots, J
\end{aligned}
$$


Compared to traditional aggregate production planning models, this formulation provides two forms of increased flexibility. The first one enables production to be less than the total demand in stage 1, therefore a constraint set such as

$$
\sum_{i=1}^{I} X_{i}=\sum_{j=1}^{J} d_{j}, \forall j=1, \ldots, J
$$

is not enforced. The second flexibility allows some of the production to be discarded in the second stage. This is accomplished by not enforcing a constraint set that all produced units are allocated to markets:

$$
\sum_{i=1}^{I} X_{i}=\sum_{j=1}^{J} \sum_{i=1}^{I} X_{i j}, \forall i=1, \ldots, I .
$$

The latter is beneficial only when the realized exchange rate is sufficiently low enough that the transportation costs cannot be justified by sales revenue from a foreign market. The following remarks are useful in understanding the value that such flexibility adds to aggregate production planning:

Remark 1 The value of $(P 1)$ with constraint sets (7) and (8) enforced is less than or equal to that of (P1) with constraint set (7) enforced.

Remark 2 The value of (P1) with constraint sets (7) and (8) enforced is less than or equal to that of (P1) with constraint set (8) enforced.

Remark 3 The value of (P1) is greater than or equal to that of (P1) with constraint set (7) enforced.

Remark 4 The value of $(P 1)$ is greater than or equal to that of $(P 1)$ with constraint set (8) enforced.

Remark 5 The value of $(P 1)$ is greater than or equal to that of $(P 1)$ with constraint sets (7) and (8) enforced.

Consequently, the optimal policy for problem (P1) is different. One optimal solution suggests that producing less than the total projected demand can be more beneficial. This means that equation (7) is not satisfied in the optimal solution, and production hedging is the prescribed optimal policy. Even if the optimal solution suggests to produce the total demand, there is no guarantee that equation (8) will be satisfied in the optimal policy. This occurs when the realized exchange rate is low enough that transportation cost is not justified by revenue times exchange rate. Therefore, the foreign market is not served, and equation (8) is not satisfied. We refer to this as allocation hedging. As a-result, the flexibility by not enforcing the equations (7) and (8), which are typically enforced in traditional modeling approaches, can provide optimal policies that employ production or allocation hedging alone, or both simultaneously. Furthermore, the set of potentially optimal solutions for the two-stage stochastic program consists of sums of some projected demand values. Since the number of solutions grows super-exponentially, it is unlikely to that a tractable computational scheme can be developed. Nevertheless, substantial understanding of the problem structure can be obtained from the analysis of special cases. 


\subsection{Impact of Exchange-Rate Uncertainty on Production Hedging}

Here, we analyze the impact of exchange-rate uncertainty in a network that consists of one plant and two markets (one domestic and one foreign). Revenue in the foreign market is collected in foreign currency. Therefore, there is a single exchange-rate variable, denoted by $e_{2}$. Decision variable $X_{1}$ represents the production quantity in the plant in the first stage, and $X_{11}$ and $X_{12}$ are the second stage allocation quantities from the plant to these two markets. Parameters $d_{1}$ and $d_{2}$ are projected demand values in the domestic and foreign countries, respectively.

To characterize the optimal solution, first observe that $P A\left(X_{1} \mid e_{2}\right)$ is the value of the optimal solution to a linear program for a given production quantity of $X_{1}$ (from stage 1) and the realized value of $e_{2}$. It is piecewise-linear and concave in $X_{1}$ with break points located at $X_{1}=\min \left(d_{1}, d_{2}\right), X_{1}=\max \left(d_{1}, d_{2}\right)$, and $X_{1}=d_{1}+d_{2}$. Since these hold true for all realizations of $e_{2}$, the first stage objective function, $E\left[P\left(X_{1}\right)\right]$, is also concave and piecewwise-linear in $X_{1}$. Hence, the optimal value of $X_{1}$ occurs at a break point. These break points are $X_{1}=0, X_{1}=\min \left(d_{1}, d_{2}\right), X_{1}=\max \left(d_{1}, d_{2}\right)$, and $X_{1}=d_{1}+d_{2} . X_{1}=0$ and $X_{1}=\min \left(d_{1}, d_{2}\right)$ can be eliminated by assumption (A.1). This leads to the following theorem:

Theorem 1 The optimal production quantity in stage 1 is either the maximum of $\left(d_{1}, d_{2}\right)$ or $d_{1}+d_{2}$.

The managerial implications of this theorem are counter-intuitive. Despite the assumption (A.1) that each market is ex-ante profitable, total production in an optimal solution may be less than the total demand. In other words, a production hedge can be optimal. Furthermore, it can be optimal to discard some production due to unfavorable exchange-rate realizations. This is termed as allocation hedging, and can be observed with or without production hedging. To develop some insight regarding allocation hedging; consider the time that the exchange rate is realized. If it is favorable enough that per unit profit (net of shipping) from the foreign market in stage 2 exceeds that of its domestic counterpart, as much output as possible is shipped to the foreign market. The remaining (if any) products are sold in the domestic market. If the exchange rate is unfavorable, however, as much output as possible is sold in the domestic market. The remaining products (if any) are shipped to the foreign market only if the revenue converted to the home country currency exceeds transportation costs; otherwise, they are discarded. Therefore, the domestic market acts as a backup that mitigates some of the risk from exchange-rate fluctuations.

Production hedging becomes optimal when there is significant exchange-rate volatility and/or when the expected profit margin is low. This is shown in the following remark:

Remark 6 The following condition, $\theta$, determines whether producing $d_{1}+d_{2}$ is preferable to producing the maximum of $\left(d_{1}, d_{2}\right)$ :

$$
\theta=\int_{\frac{c_{12}}{r_{2}}}^{\frac{r_{1}+c_{12}}{r_{2}}}\left(r_{2} e_{2}-c_{12}\right) f\left(e_{2}\right) d e_{2}+\int_{\frac{r_{1}+c_{12}}{r_{2}}}^{\infty}\left(r_{1}\right) f\left(e_{2}\right) d e_{2}-c_{1} \geq 0
$$


and the value of $\theta$ increases with increasing values of $r_{1}$ and $r_{2}$ and decreasing values of $c_{1}$ and $c_{12}$. Therefore,

a) $\frac{\partial \theta}{\partial r_{1}}>0$

b) $\frac{\partial \theta}{\partial r_{2}}>0$

c) $\frac{\partial \theta}{\partial c_{1}}<0$

d) $\frac{\partial \theta}{\partial c_{12}}<0$.

We already know from Theorem 1 that the firm must produce at least the maximum of $\left(d_{1}, d_{2}\right)$. Because of linearity, $\theta$ can be interpreted as a derivative that represents the value of producing one more unit beyond the maximum of $\left(d_{1}, d_{2}\right)$. The cost of producing one more unit is $c_{1}$. The two integral terms in (9) represent the expected second stage return from a unit increase in production. Each term has the returns from the less desired market as a result of this marginal perspective. Accounting for all contingencies, the first integral represents the incremental revenue which results from using this unit to serve the foreign market. If the exchange rate is unfavorable (when $e_{2}-c_{12} / r_{2}<0$ ) this incremental unit is not used. This is more likely to occur with a higher value of $c_{12}$, the unit transportation cost, relative to the unit revenue in the foreign market. The same conclusion is made when the exchange rate distribution is volatile and has a higher mass between the values of 0 and $c_{12} / r_{2}$. The second integral term has a constant return, $r_{1}$; for all realizations of $e_{2}$. This term represents the concurrent incremental revenue from being able to sell one more unit in the domestic market. As a result, if the total incremental revenue exceeds the unit production cost, $c_{1}, \theta$ is non-negative, and it is beneficial to produce one more unit. Then, it follows from linearity that it is best to increase production to the total demand. Otherwise, it is optimal to produce the maximum of $\left(d_{1}, d_{2}\right)$; in other words, follow a production hedging policy. As can be summarized from Remark 6, whenever the expected profit margin is low or there is higher volatility in exchange rates, the more likely it is that $\theta$ will be negative; consequently the more likely it is that production hedging will be optimal.

Suppose that (9) is not satisfied and it is optimal to follow a production hedging policy; in other words produce less than the total demand. When this is the case managers may be uncomfortable with deliberately underserving markets. $\theta$ offers insight as to how a firm can remedy this. From an operations perspective, the firm could lower the unit manufacturing $\operatorname{cost} c_{1}$ and/or the unit transportation cost $c_{12}$ making $\theta$ larger. Such action is solely within the domain of the firm and has the added effect of raising the profit margins for all. customers. From a management perspective, the firm could attempt to raise the price in one or both markets to make $\theta$ larger. The latter would be considerably more difficult to carry out because of internal transfer pricing policies and external price competition.

Even if management can satisfy (9) so that it is optimal to produce the total demand, there is no guarantee that both markets will be served. This is due to the fact that under allocation hedging production will be discarded when exchange rates are unfavorable. An obvious mechanism to overcome this adverse action is to buy a call option to limit downside risk. We next calculate the value of purchasing such financial instruments 
in the context of this problem. This economic value enables managers to make interactive decisions between financial and operational hedging.

\subsection{Economic Values of Financial and Operational Hedging and Their Interaction}

We now determine how much value financial hedging would add to the global production planning problem. Because our model has the flexibility of not satisfying equations (7) and (8), the decision maker has to consider the interaction between financial and operational hedging. The value calculated here corresponds to the manager's reservation price to employ a financial instrument such as a call option under various operational hedging schemes. The reservation price is the maximum that the decision maker would be willing to pay for such an instrument. To simplify the exposition, we assume that both markets have identical demand $d$. The incremental value of utilizing financial hedging is shown for both of the potentially optimal policies, production hedging, and producing the total demand. First, consider the case where the optimal solution is a production hedge which suggests manufacturing only $d$. However, the firm is interested in making certain that total production is $2 d$ and that both markets will be fully served - namely, neither production nor allocation hedging will be exercised. Clearly this solution is sub-optimal and riskier to the firm. Notice that this risk derives from converting the revenue of $r_{2} e_{2}$ into the numeraire currency. An obvious way to mitigate this risk is to purchase a call option to convert $r_{2} d$ units of the foreign currency at the numeraire amount of $r_{2} e_{2}^{s} d$ (strike price of $e_{2}^{s}$ ). The premium paid to purchase this option is ignored for the time being in order to identify ex-post returns. However, it is used to compare the value-added to the system at the end, and the decision to purchase the option depends on this comparison.

This simple call option, as shown in the Appendix, has a positive value if the strike price is greater than or equal to the ratio of the sum of unit manufacturing and transportation costs to the unit revenue from the foreign market, $e_{2}^{s} \geq \frac{c_{1}+c_{12}}{r_{2}}$. Its value to the firm is greater than $r_{1}-c_{1}$ if the foreign customer is more profitable ex-ante $\left(r_{1}<r_{2} e_{2}^{s}-c_{12}\right)$. Its value is between zero and $r_{1}-c_{1}$ if the foreign customer is less profitable ex-ante $\left(r_{1} \geq r_{2} e_{2}^{s}-c_{12}\right)$. The incremental value of utilizing such an option, denoted as $V\left(e_{2}^{s}\right)$, when producing and satisfying all requirements can be written as follows:

$$
V\left(e_{2}^{s}\right)=\left\{\begin{array}{c}
d\left[r_{1}-c_{1}+\int_{0}^{\left(r_{1}+c_{12}\right) / r_{2}}\left(r_{2} e_{2}^{s}-\dot{c}_{12}-r_{1}\right) f\left(e_{2}\right) d e_{2}+\int_{\left(r_{1}+c_{12}\right) / r_{2}}^{e_{s}^{s}} r_{2}\left(e_{2}^{s}-e_{2}\right) f\left(e_{2}\right) d e_{2}\right] \\
\text { if } r_{1}<r_{2} e_{2}^{s}-c_{12} \\
d\left[r_{1}-c_{1}+\int_{0}^{e_{2}^{s}}\left(r_{2} e_{2}^{s}-c_{12}-r_{1}\right) f\left(e_{2}\right) d e_{2}+\int_{e_{2}^{s}}^{\left(r_{1}+c_{12}\right) / r_{2}}\left(r_{2} e_{2}-c_{12}-r_{1}\right) f\left(e_{2}\right) d e_{2}\right] \\
\text { if } r_{1} \geq r_{2} e_{2}^{s}-c_{12}
\end{array}\right\}
$$

Notice that each expression for $V\left(e_{2}^{s}\right)$ is similar to that of $\theta$; however, in the region of allocation hedging, $0 \leq e_{2} \leq c_{12} / r_{2}, V\left(e_{2}^{s}\right)$ is always positive. Notice that it is 0 in the same region in $\theta$. Hence, we can conclude 
that if a call option to convert $r_{2} d$ units of the foreign currency at the strike price of $e_{2}^{s}$ can be purchased for a premium of up to $V\left(e_{2}^{s}\right)$, then it is optimal to increase production to the total demand and fully serve both customers. A similar approach can be used to show that if the optimal policy were to produce the total demand, and a call option were to be purchased to hedge against the exchange-rate risk, it too would have a positive value. Since a call option with a strike price, $e_{2}^{s}$, greater than or equal to $c_{12} / r_{2}$ prevents allocation hedging, the incremental value can be written as:

$$
V\left(e_{2}^{s}\right)=d\left[\int_{0}^{c_{12} / r_{2}}\left(r_{2} e_{2}^{s}-c_{12}\right) f\left(e_{2}\right) d e_{2}+\int_{c_{12} / r_{2}}^{e_{2}^{s}} r_{2}\left(e_{2}^{s}-e_{2}\right) f\left(e_{2}\right) d e_{2}\right]
$$

Similarly, if a call option can be purchased for a premium up to this value, then it is optimal to purchase the financial option and guarantee serving both markets. Otherwise, it is still optimal to produce the total demand, however, the possibility of allocation hedging is not eliminated.

We have outlined how the presence of exchange-rate uncertainty necessitates a cross-functional approach to overcoming its adverse effects when engaging in production planning for a global manufacturing network. The structural results described above are quite robust, in the sense that they hold for any exchange-rate distribution.

The model used in this section considered one foreign market and explored only the effect of exchange rate volatility. It can be shown that the policy implications of our model are valid in the presence of correlated exchange rates among foreign markets and provides additional insight regarding their impact on choices.

\subsection{Impact of Correlated Exchange Rates}

It is not necessary for a domestic market to be present in order for a production hedge to be effective. We now examine how the policy structure changes when there is one plant and two foreign markets whose exchange rates are correlated. Define $r_{2}$ and $r_{3}$ as the unit revenues from selling in countries 2 and 3 , respectively. $X_{1}$ (first stage decision variable) is the amount of production in the plant, and $X_{12}$ and $X_{13}$ (second stage decision variables) are the quantities shipped to the second and third markets, respectively. To simplify the exposition, we assume that the demand in each country is equal to $d$. Since both markets are foreign, two exchange rates must be considered. $e_{2}$ and $e_{3}$ denote the two random variables that represent these exchange rates. These two random variables have a joint pdf $f\left(e_{2}, e_{3}\right)$; the correlation coefficient is $\rho(-1 \leq \rho \leq+1)$. In this case, $\rho$ plays a crucial role in determining how much to produce. Production hedging is apt to occur when $\rho$ is sufficiently small. We begin the analysis with the following:

Theorem 2 The optimal production quantity in stage 1 is either $d$ or $2 d$.

While Theorem 2 is useful in characterizing the structure of the optimal policy, it is also of interest to examine the sensitivity of the optimal solution to $\rho$, the correlation between two exchange rates. We begin by 
defining the function $\delta(\rho)$ as the difference between the expected profits of producing $2 d$ and $d$ for a given $\rho$.

$$
\delta(\rho)=E P\left[X_{1}=2 d \mid \rho\right]-E P\left[X_{1}=d \mid \rho\right]
$$

It follows that when $\delta(\rho)$ is negative, the optimal production quantity is $d$, so that the optimal solution can be interpreted as a production hedging policy. Conversely, when $\delta(\rho)$ is positive, the optimal production quantity is $2 d$. To make the optimal production decision, it is sufficient to evaluate the sign of $\delta(\rho)$. Although, in general, the evaluation of $\delta(\rho)$ depends on specifying a joint density for $e_{2}$ and $e_{3}$, there are important exceptions. It should be noted here that the line $r_{2} \overline{e_{2}}-c_{12}=r_{3} \overline{e_{3}}-c_{13}$ divides the exchange rate space diagonally into two sections. Considering that the vertical axis represents $e_{2}$ values, a realization of exchange rate below this line makes the second stage return of $r_{3} e_{3}-c_{13}$ higher than $r_{2} e_{2}-c_{12}$. Therefore revenue from foreign market 3 is preferred over that of foreign market 2. It can be shown that $\delta(\rho)$ is always positive if the density is positive only above the choice line of $r_{2} \overline{e_{2}}-c_{12}=r_{3} \overline{e_{3}}-c_{13}$ or only below $r_{2} \overline{e_{2}}-c_{12}=r_{3} \overline{e_{3}}-c_{13}$. When this is the case, the optimal policy is to produce $2 d$.

In general, however, the joint density will be positive on both sides of the choice line, $r_{2} \overline{e_{2}}-c_{12}=r_{3} \overline{e_{3}}-c_{13}$, thus additional analysis of $\delta(\rho)$ is warranted. Intuition suggests that if the production hedging policy $X_{1}=d$ is optimal $(\delta(\rho)<0)$ it is more apt to occur when $\rho$ is close to -1 . This is due to the fact that a favorable value of $e_{2}$ is balanced by an unfavorable value of $e_{3}$. As $\rho$ increases this balance would tilt towards full production $(\delta(\rho)>0)$. In order to further examine the behavior of $\delta(\rho)$, some restrictions need to be imposed on the density. Adequate tractability is achieved, while providing a rich modeling framework, by restricting the density to the following sub-family of the family of densities developed in Van Mieghem [1995]:

$$
\begin{aligned}
& f\left(e_{2}, e_{3} \quad \mid \quad \rho\right)=\left\{\begin{array}{lll}
\frac{1}{\pi \sqrt{1-\rho^{2}}} & \text { if }\left(\frac{e_{2}-\overline{e_{2}}}{2 \sigma_{2}}\right)^{2}-2 \rho\left(\frac{e_{2}-\overline{e_{2}}}{2 \sigma_{2}}\right)\left(\frac{e_{3}-\overline{e_{3}}}{2 \sigma_{3}}\right)+\left(\frac{e_{3}-\overline{e_{3}}}{2 \sigma_{3}}\right)^{2}<1-\rho^{2} \\
0 & \text { otherwise } & \text { for }-1<\rho<+1
\end{array}\right\} \\
& =\left\{\begin{array}{ccc}
\frac{1}{2 \sqrt{2}} & \text { if }\left(\frac{e_{2}-\overline{e_{2}}}{2 \sigma_{2}}\right)=-\left(\frac{e_{3}-\overline{e_{3}}}{2 \sigma_{3}}\right) \text { and }-1 \leq\left(\frac{e_{1}-\overline{e_{2}}}{2 \sigma_{2}}\right) \leq+1 \text { and }-1 \leq\left(\frac{e_{3}-\overline{e_{3}}}{2 \sigma_{3}}\right) \leq+1 \\
0 & \text { otherwise } & \text { for } \rho=-1
\end{array}\right\} \\
& =\left\{\begin{array}{lll}
\frac{1}{2 \sqrt{2}} & \text { if }\left(\frac{e_{2}-\overline{e_{2}}}{2 \sigma_{2}}\right)=\left(\frac{e_{3}-\overline{e_{3}}}{2 \sigma_{3}}\right) \text { and }-1 \leq\left(\frac{e_{2}-\overline{e_{3}}}{2 \sigma_{2}}\right) \leq+1 \text { and }-1 \leq\left(\frac{e_{3}-\overline{e_{3}}}{2 \sigma_{3}}\right) \leq+1 \\
0 & \text { otherwise } & \text { for } \rho=+1
\end{array}\right\}
\end{aligned}
$$

Notice that in (13) the density is non-negative and uniform on an ellipse when $-1<\rho<+1$, and on a line-segment when $\rho=-1$ or $\rho=+1$. Next, we show that $\delta(\rho)$ is monotonically increasing with increasing values of $\rho$. Using a common practice of setting prices to make both markets equally attractive ex-ante, or $r_{2} \overline{e_{2}}-c_{12}=r_{3} \overline{e_{3}}-c_{13}$, we can prove the following: 
Theorem 3 If $r_{2} \overline{e_{2}}-c_{12}=r_{3} \overline{e_{3}}-c_{13}$ and $f\left(e_{2}, e_{3} \mid \rho\right)$ is given by (13), then $\delta(\rho)$ is monotonically increasing with the increasing values of $\rho$.

The implication of Theorem 3 is that there exists at most one solution to $\delta(\rho)=0$. We denote the correlation coefficient that satisfies $\delta(\rho)=0$ as $\rho^{*}$. The value of this critical correlation coefficient can be found in the following theorem.

Theorem 4 If $r_{2} \overline{e_{2}}-c_{12}=r_{3} \overline{e_{3}}-c_{13}$ and $f\left(e_{2}, e_{3} \mid \rho\right)$ is given by (13), then the critical correlation coefficient, $\rho^{*}$, that satisfies $\delta(\rho)=0$ is equal to:

$$
\rho^{*}=\frac{1}{2}\left(\frac{r_{2} \sigma_{2}}{r_{3} \sigma_{3}}\right)+\frac{1}{2}\left(\frac{r_{3} \sigma_{3}}{r_{2} \sigma_{2}}\right)-\frac{9 \pi^{2}}{32} \frac{\left(r_{2} \overline{e_{2}}-c_{12}-c_{1}\right)}{r_{2} \sigma_{2}} \frac{\left(r_{3} \overline{e_{3}}-c_{13}-c_{1}\right)}{r_{3} \sigma_{3}}
$$

when $-1<\frac{1}{2}\left(\frac{r_{2} \sigma_{2}}{r_{3} \sigma_{3}}\right)+\frac{1}{2}\left(\frac{r_{3} \sigma_{3}}{r_{2} \sigma_{2}}\right)-\frac{9 \pi^{2}}{32} \frac{\left(r_{2} \overline{e_{2}}-c_{12}-c_{1}\right)}{r_{2} \sigma_{2}} \frac{\left(r_{3} \overline{e_{3}}-c_{13}-c_{1}\right)}{r_{3} \sigma_{3}}<+1$.

When the correlation coefficient among two exchange rates is below $\rho^{*}$, then production hedging is the optimal solution; otherwise, it is more profitable to produce the total demand. The critical correlation coefficient can also be seen as the point where the company switches its policy from production hedging to satisfying the total demand. In order for the company to satisfy the total demand, it has to have either a sufficiently high profit margin or a relatively low volatility in exchange rates. It is useful to observe that the first 2 terms of (14) balance reciprocally the relative risk from the two markets, while the last term adjusts for the joint risk from the two markets.

Remark 7 The critical correlation coefficient, $\rho^{*}$, of (14) decreases with

a) increasing values of expected second stage contributions, i.e. $r_{2} \overline{e_{2}}-c_{12}$

b) decreasing values of standard deviation of exchange rates, $\sigma_{2}$ and $\sigma_{3}$

Knowing that managers would like to satisfy total demand, it seems that it would be in their best interest to increase local prices. Although this may not be possible due to competition, assuming that it can be increased up to infinity provides a threshold value for the optimal solution. In the limit when $r_{2}$ and $r_{3}$ go to infinity, production hedging is still optimal when the correlation coefficient among two exchange rates is below this threshold value. We use $c v_{2}\left(c v_{2}=\frac{\sigma_{2}}{e_{2}}\right)$ and $c v_{3}\left(c v_{3}=\frac{\sigma_{3}}{e_{3}}\right)$ to denote the coefficient of variation for exchange rates of $e_{2}$ and $e_{3}$, respectively.

Theorem 5 When $r_{2} \overline{e_{2}}-c_{12}=r_{3} \overline{e_{3}}-c_{13}$ and $f\left(e_{2}, e_{3} \cdot \rho\right)$ is given by (13), production hedging is always optimal for correlation coefficient values that are smaller than or equal to:

$$
\frac{1}{2}\left(\frac{\sigma_{2}}{\sigma_{3}}\right)+\frac{1}{2}\left(\frac{\sigma_{3}}{\sigma_{2}}\right)-\frac{9 \pi^{2}}{32} \frac{1}{c v_{2} c v_{3}}
$$

when $-1<\frac{1}{2}\left(\frac{\sigma_{2}}{\sigma_{3}}\right)+\frac{1}{2}\left(\frac{\sigma_{3}}{\sigma_{2}}\right)-\frac{9 \pi^{2}}{32} \frac{1}{c v_{2} c v_{3}}<+1$.

When $\sigma_{2}=\sigma_{3}$ is assumed, the above threshold simplifies to $1-\frac{9 \pi^{2}}{32} \frac{1}{c v_{2}^{2}}$. Therefore, under this assumption, production hedging is always optimal when $c v_{2} \geq \frac{3 \pi}{4 \sqrt{2}}$. 


\subsection{Effect of Exchange Rate Realization Timing}

We now consider the timing effect of exchange-rate realization on the optimal production and allocation policies. As discussed in Section 1, the recourse problem is sensitive to the timing of exchange-rate realizations. Interestingly, not only is expected profit monotone in the timing of information, but as we show for the one plant, one domestic and one foreign market case, so is the optimal policy. As a general rule, the later exchange rates are realized, the lower is the expected profit and the higher is the investment in capacity. We begin with a comparison of the recourse-after-production model with the recourse-after-capacity model. The only difference between these two models is that the exchange rate is observed after capacity commitment but before production commitment occurs in the recourse-after-capacity model; it is observed after production in the recourse-after-production model. In the recourse-after-production model, $c_{1}$ is the unit cost of manufacturing and capacity investment in the plant. If $c_{1}$ is decomposed into two terms as the unit cost of manufacturing, defined as $c_{m}$, and the unit cost of capacity investment, defined as $c_{c}$, where $c_{1}=c_{m}+c_{c}$, these two models become directly comparable. Capacity investment occurs prior to production in both models. The unit cost of manufacturing, however, occurs in the second stage in the recourse-after-capacity model whereas it occurs in the first stage in the recourse-after-production model. Thus for the same capacity investment, the expected profit is never lower in the recourse-after-capacity model. However, when the capacity is less than the minimum requirement of the two markets, the expected profit for each of these models is identical and increases with the same slope. When capacity increases beyond this minimum, the unit contribution to expected profit is higher in the recourse-after-capacity model as illustrated in Figure 2.

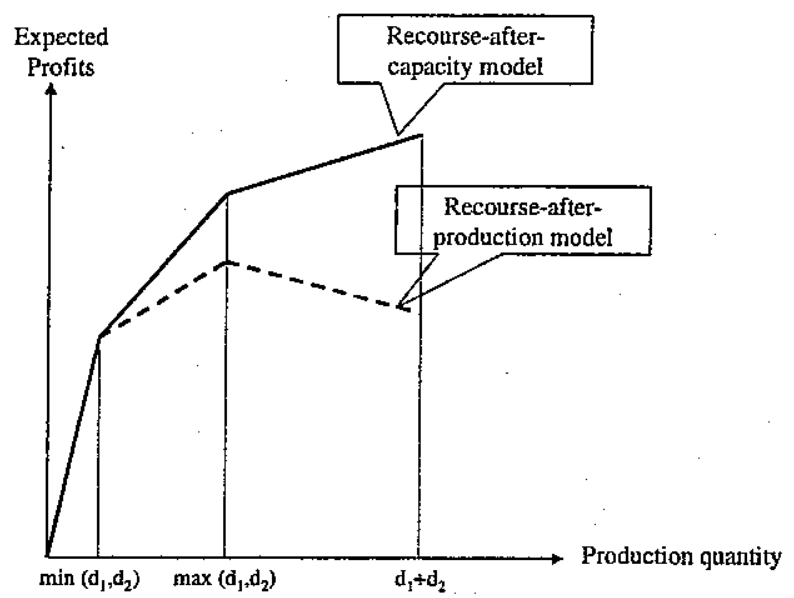

Figure 2: A sample of expected profit vs. production quantity graphs for a recourse-after-capacity and a recourse-after-production model 
Because the exchange rate is observed prior to the production and allocation decisions, the recourse-aftercapacity model has neither allocation nor production hedging. Instead it has a capacity hedge because the capacity decision can be less than the total demand. It can also experience an unfavorable recourse of capacity because production can be less than capacity. These insights are formalized as:

Theorem 6 Expected profit from the optimal production policy in the recourse-after-capacity model is always greater than or equal to that of the recourse-after-production model.

The single-period version of the model of Huchzermeier and Cohen [1996] is a form of the recourse-aftercapacity model. In their multi-period model they commit to capacity before the start of each period, and after observing the exchange rate they decide on production and allocation decisions. Since their capacity commitment is in lumpy quantities it is unlikely that structural results like those in our model can be obtained. They conclude that the firm would engage in higher capacity investment under exchange-rate uncertainty. This can be seen as a capacity hedging policy that mitigates the downside risk of exchange-rate uncertainty.

The following theorem allows us to compare the results of the recourse-after-capacity model with the recourseafter-production model. This theorem states that the single-period version of the recourse-after-capacity model has structural results similar to the recourse-after-production model. Furthermore, in the single-period version, the optimal capacity investment does not exceed the total demand. We conjecture that the recommendation of Huchzermeier and Cohen [1996], i.e. investing in higher capacity to compensate for exchange-rate uncertainty, is a consequence of the fact that their capacity choices are discrete.

Theorem 7 a) If the optimal production in the recourse-after-production model is the total demand, $d_{1}+d_{2}$, then in the recourse-after-capacity model the optimal capacity investment is also equal to the total demand, $d_{\mathrm{I}}+d_{2}$; b) If the recourse-after-capacity model results in a capacity hedging policy (investing in less capacity than the total demand), then the recourse-after-production model also prescribes production hedging in optimal solutions.

This theorem does not imply that the two models always provide the same results, but that $X_{1}$ in the recourseafter-capacity model is never less than the corresponding value in the recourse-after-production model. Indeed, there might be cases where the recourse-after-capacity model yields a capacity investment equal to the total demand whereas the recourse-after-production model calls for a production hedging policy. This result is due to the timing of exchange-rate realization. The optimal policy for these models may recommend completely different sets of capacity investment, production quantity, and allocation to market decisions. Therefore, we conclude that the timing of exchange-rate realization influences all operational decisions; namely capacity investment, production planning, and allocation of production to markets.

In both models a hedge can occur in two ways: (a) the first is the case where the profit margin is small and/or the exchange rate distribution is volatile, and (b) the second is where there is a significant amount of 
allocation hedging. It should be noted that allocation hedging is a consequence of high transportation cost relative to the price. Analogous results can be obtained by a pairwise comparison of the perfect information model to the recourse-after-capacity model; and to the recourse-after-production model with the no-information model.

\section{An Empirical Study of a Global Manufacturing Network}

This section demonstrates how our model may be employed to make aggregate planning decisions in an actual empirical setting. The data is adapted from a global manufacturing network of IBM. Our objective is not to assess network performance, but rather to illustrate the application of our model and the potential benefits that could result from using it. Although the actual network is quite complex, for illustrative purposes we can consider it to consist of three regions: North America, Europe, and Asia/Pacific. Each of these three markets has a projected demand and only two of these three markets have a production plant. We employ a sample of daily real exchange-rate quotations from September 1996 to February 1997, as recorded in International Financial Statistics. The data set has 115 observations and the following statistical properties: $\mu_{D M}=0.640917 ; \mu_{Y}=$ $0.008724 ; \sigma_{D M}=0.025190 ; \sigma_{Y}=0.000338 ; \rho=0.96119$. Here $\mu_{D M}$ and $\mu_{Y}$ are the mean, and $\sigma_{D M}$ and $\sigma_{Y}$ are the standard deviations of the Deutsche Mark and the Japanese Yen, respectively. The price in Europe is expressed in Deutsche Marks (DM), for Asia/Pacific the Japanese Yen, and for North America the US Dollar. It is important to note that in this specific period of time which the data set represents, exchange rates of these currencies did not exhibit great volatility.

One approach to solving this problem is to fit a joint distribution to the underlying exchange rates and then attempt to directly solve the resulting stochastic program. Alternatively, as done here, we can characterize admissible extreme points (there are 23). If we think of each of these solutions as a scenario that is feasible in the first stage, when the expected value of the second stage program is known, these 23 scenarios could then be compared to determine the optimal solution. Rather than compute the expectation, we generate an estimate of it by solving the second-stage linear programs for each of the 115 exchange rate observations. The mean of each of these 115 solutions net of production costs yields an unbiased estimate of the expected profit for that scenario. The scenario yielding the highest expected profit is then selected as the optimal solution.

Our numerical experiment reveals that the optimal solution for this product is to produce the total demand. Moreover, exchange rates are sufficiently stable so that allocation hedging is never exercised; consequently all three markets are fully served. The same analysis is replicated with prices that are $3.6 \%$ lower in each market. (The earlier assumptions on ex-ante profitability are not violated with the reduction in prices.) In contrast to the case with original prices, the optimal solution is a production hedging policy that calls for producing just $50 \%$ of the total demand; once again allocation hedging is not exercised. It is interesting to note that the 
expected profit under this optimal production hedging policy is $137 \%$ higher than under the best plan that produces the total demand. This result highlights the importance of exchange rate volatility on production planning. The benefits of using production hedging under significant volatility are likely to be even greater.

Since the expected profit under the production hedge is significantly higher, and, a strategic decision is made to serve all markets, the ensuing financial risk can be mitigated by buying call options with the Deutsche Mark and Yen as discussed in Section 3.3. The strike prices of each of these options are equal to its mean value. Recall that when such options are utilized, the optimal solution is to produce the total demand and satisfy all markets. In order to simplify the comparison, we rescale expected profits by taking the best solution of producing the total demand with no financial options as the basis $(\$ 1.00)$. With this scale, the optimal production hedging solution has an expected profit of $\$ 2.37$. The optimal expected profit using financial options is $\$ 2.70,170 \%$ higher than the base case plan when the premium for these options are excluded. This expected profit is also $13.77 \%$ higher than in the optimal production hedging policy. From an operations manager's perspective, this difference of $\$ 0.33$ corresponds to the value of using such options. Alternatively, it determines her/his reservation price. If the premium of the options costs less than that $\$ 0.33$, then it is beneficial to buy the options, produce the total demand, and satisfy all markets. Suppose that the premium is higher than $\$ 0.33$ but less than $\$ 1.70$. In contrast, if the corporate policy is to fully serve all markets as part of their strategic plans, then the reservation price from a corporate perspective is $\$ 1.70$. Hence, we can conclude that up to $\$ 0.33$ of the premium should be charged to the operations manager, and the remaining premium should be absorbed at the corporate level. This "realistic". example not only shows that the value of using financial instruments can be substantial, it also provides insight on how to share the expenses of such an action among production divisions and corporate headquarters.

\section{Conclusions and Managerial Insights}

We have investigated the impact of exchange-rate uncertainty on production planning decisions in a global manufacturing network. A two-stage stochastic program is developed in which the firm is given the choice of serving less than the market demand. This is accomplished ex-ante by a "production hedge" under which total production is less than total demand. It is exercised ex-post by not allocating all production to markets when the exchange rates are too unfavorable. The latter option is termed "allocation hedging."

We began our analysis by identifying the set of potentially optimal production policies and the conditions which enforce them. When the production hedging policy is optimal, we calculate the reservation price for a manager who wants to utilize a financial instrument, e.g. an option, in order to mitigate the downside risk of exchange-rate fluctuations and of satisfying demand in all markets. It is important to note that no assumptions were made with regard to exchange-rate distributions, thus these structural results are robust. By using a 
special family of distributions (Van Mieghem [1995]), we are able to show that similar structural results hold when there are multiple exchange rates that are correlated. Finally, our analytical results are illustrated with actual data from a global manufacturing network of IBM that reveals the substantial benefits of production and financial hedging.

The analysis presented yields three main results. First, notwithstanding the assumption that each market is ex-ante profitable we show that under significant exchange-rate risk it can be optimal to produce less than the total demand. We refer to this as the "production hedging" policy. Several examples of this policy can be observed in industrial practice. For example, an article in the Wall Street Journal ("Weak Yen Stymies U.S. Strategies for Asia," June 15, 1998, pp. A2) states that the Chrysler Corp. recognizes that there is unmet demand for its U.S. made cars in Japan, but, the company is not quite sure whether to explore this market due to the downside risk of the Japanese Yen: "In December, when a dollar sold for around 130 yen, Chrysler Corp. chief economist M. Van Bussman warned that if the U.S. currency rose to 140 yen, 'it would scare the pants off us.' On Friday, a dollar bought 144.33 yen in New York." The article concludes, "That puts quite a chill on Chrysler's efforts to sell cars in Japan." Despite the demand in Japan, Chrysler chooses not to serve the Japanese market due to the fall of the yen.

The second result shows that production hedging is more likely to be optimal when the profit margin is small and/or the exchange rate is highly volatile. In order to provide further insight on this, we analyze the case of correlated multiple exchange rates using a sub-family of the family of densities developed by Van Mieghem [1995]. This yields a third result that the probability of following a production hedging policy as an the optimal solution decreases as the correlation coefficient between exchange rates monotonically increases from being perfectly negatively correlated to being perfectly positively correlated. We then find a critical correlation coefficient that determines when to switch from a policy of production hedging to producing the total demand. The value of this "critical" correlation coefficient is impacted by the profit margin and the exchange rate coefficient of variation. These results provide managerial insight and guidance by incorporating the two main factors that influence risk in exchange-rate markets: volatility of each exchange rate and their correlation.

Our model also suggests that when exchange rates exhibit high volatility and the optimal solution calls for a production hedge, we show how the plan can be modified to fully serve all markets. This is achieved by developing an integrated solution that requires collaborative decision making among three different functional areas (marketing, operations, and finance.) From a marketing perspective, the possibility of increasing prices can be explored (however, this may not be easily accomplished due to external price competition or internal transfer pricing policy); or, reducing manufacturing costs can be considered by the operations function. Finally, the feasibility of utilizing a financial instrument to mitigate the downside risk can be investigated. Indeed, such approaches are used in practice. For example, in another article in the Wall Street Journal ("UTC Battles Asian Slump with Cutback," August 19, 1998, pp. A1, A6), it was reported that the United Technologies Corp. 
(UTC - producer of heating/air conditioning instruments and elevators) chose to make all efforts in reducing manufacturing costs as it could not increase prices due to competition. The article also stated that UTC was decreasing its capacity investment in Asia due to fluctuations in exchange rates. This decision was made despite the fact that Asia was the fastest growing market for its products. This corresponds to a form of production hedging.

Finally, we propose three extensions for future research. The first is to develop models that consider inventory holding decisions in a multi-period context. We conjecture that policies such as production hedging are likely to be optimal in this setting as well. The second extension is to examine the combined impact of exchange rate and demand uncertainty on production and capacity planning decisions. In view of the above studies, the third extension would consider more strategic decisions such as the number and location of facilities.

\section{Acknowledgments}

We would like to thank Dr. Robert Baseman and Mr. William Grey of the IBM T. J. Watson Research Center, Yorktown Heights, NY for the insight they provided during numerous enlightening conversations. We are also grateful for their generous contribution of data for sample IBM products.

\section{References}

[1] Christopher, M., "Logistics and Supply Chain Management," IRWIN Professional Publishing, New York, 1994.

[2] Cohen, M., Huchzermeier, A., "Global Supply Chain Management: A Survey of Research and Applications," pp. 669-702, edited by S. Tayur et al., Kluwer Academic Publishers, Massachusetts, 1999.

[3] Dasu, S., Li, L., "Optimal Operating Policies in the Presence of Exchange Rate Variability," Management Science, Vol. 43, No. 5, pp. 705-722, 1997.

[4] Flaherty, T., "Global Operations Management," McGraw Hill, New York, 1996.

[5] Hodder, J., "Financial Market Approaches to Facility Location under Uncertainty," Operations Research, Vol. 32, pp. 1374-1380, 1984.

[6] Hodder, J., Jucker, J., "International Plant Location under Price and Exchange Rate Uncertainty," Engineering Costs and Production Economics, Vol. 9, pp. 225-229, 1985a.

[7] Hodder, J., Jucker, J., "A Simple Plant Location Model for Quantity-Setting Firms Subject to Price Uncertainty," European Journal of Operations Research, Vol. 21, pp. 39-46, $1985 \mathrm{~b}$.

[8] Hodder, J., Dincer, C., "A Multifactor Model for International Plant Location and Financing under Uncertainty," Computers and Operations Research, Vol. 13, No. 5, pp. 601-609, 1986. 
[9] Huchzermeier, A., "Evaluating the Benefit of Operational Flexibility and the Real Option Value of Managerial Flexibility in Global Supply Chain Networks," Production and Operations Management (forthcoming), 1998.

[10] Huchzermeier, A., Cohen, M., "Valuing Operational Flexibility Under Exchange Rate Risk," Operations Research, Vol. 44, pp. 100-113, 1996.

[11] Jucker, J., Carlson, R., "The Simple Plant-Location Problem under Uncertainty," Operations Research, Vol. 24, pp. 1045-1055, 1976.

[12] Kogut, B., Kulatilaka, N., "Operating Flexibility, Global Manufacturing, and the Option Value of a Multinational Network," Management Science, Vol. 40, No. 1, pp. 123-139, 1994.

[13] Kouvelis, P., "Global Sourcing Strategies under Exchange Rate Uncertainty, " pp. 625-667, edited by S. Tayur et al., Kluwer Academic Publishers, Massachusetts, 1999.

[14] Kouvelis, P., Axarloglou, K., Sinha, V., "Exchange Rates and the Choice of Ownership Structure of Production Facilities," Management Science, Vol. 47, No. 8, pp. 1063-1080, 2001.

[15] Kouvelis, P., Gutierrez, G., "The Newsvendor Problem in a Global Market: Optimal Centralized and Decentralized Control Policies for a Two-Market Stochastic Inventory System," Management Science, Vol. 43, No. 5, pp. 571-585, 1997.

[16] Petruzzi, N., Dada, M., "Information and Inventory Recourse for a Two-Market, Price-Setting Retailer," Working Paper, 2000.

[17] Rosenfield, D. B., "Global and Variable Cost Manufacturing Systems," European Journal of Operations Research, Vol. 95, pp. 325-343, 1996.

[18] Scheller-Wolf, A., Tayur, S., "Managing Supply Chains in Emerging Markets," pp. 703-735, edited by S. Tayur et al., Kluwer Academic Publishers, Massachusetts, 1999.

[19] Van Mieghem, J. A., "Multi-Resource Investment Strategies under Uncertainty," Unpublished Doctoral Dissertation, Graduate School of Business, Stanford University, 1-89, May 1995. 